\title{
Incremental Perspective Motion Model for Rigid and Non-rigid Motion Separation
}

\author{
Tzung-Heng Lai, Te-Hsun Wang, and Jenn-Jier James Lien \\ Robotics Laboratory, CSIE, NCKU, Tainan, Taiwan, R.O.C. \\ \{henry, dsw_1216, jjlien\}@csie.ncku.edu.tw
}

\begin{abstract}
Motion extraction is an essential work in facial expression analysis because facial expression usually experiences rigid head rotation and non-rigid facial expression simultaneously. We developed a system to separate non-rigid motion from large rigid motion over an image sequence based on the incremental perspective motion model. Since the parameters of this motion model are able to not only represnt the global rigid motion but also localize the non-rigid motion, thus this motion model overcomes the limitations of existing methods, the affine model and the 8-parameter perspective projection model, in large head rotation angles. In addition, since the gradient descent approach is susceptible to local minimum during the motion parameter estimation process, a multi-resolution approach is applied to optimize initial values of parameters at the coarse level. Finally, the experimental result shows that our model has promising performance of separating non-rigid motion from rigid motion.
\end{abstract}

Keywords: Separating rigid and non-rigid motion, incremental perspective motion model, multi-resolution approach.

\section{Introduction}

Computer vision researchers have developed various techniques on automatic facial expression recognition. Some exiting recognition systems [10], [11], [13], [14], [16] apply the facial images without taking rigid and non-rigid motion separation process; and the tolerance of rotation angles are not mentioned. Work in [2] uses Adaboost for feature selection, and then classifying the selected outputs by the support vector machine (SVM). The combination of Adaboost and SVM enhanced both speed and accuracy of recognition system. The study in [17] integrated the dynamic Bayesian networks (DBNs) with the facial action units for modeling the dynamic and stochastic behaviors of expressions. $\pm 30^{\circ}$ out-of-plane rotations are allowed in their tracking technique that one active IR illumination is used to provide reliable visual information under variable lightings and head motions. The work in [1] used SVM to classify the facial motions for recognizing six basic emotions associated with unique expressions i.e. happiness, sadness, disgust, surprise, fear and anger. The limitations of head rotation angles are $\pm 30^{\circ}$ in pan rotation, $\pm 20^{\circ}$ in tilt rotation and $\pm 10^{\circ}$ in roll rotation. The work in [7] also used SVM to classify five facial motions, neutral expression, opening or closing mouth, smile and raising eyebrows, with one deformable model 
containing 19 points and stereo system. The stereo tracking serves as the basis for reconstructing the subject's 3D face model with deformable model and constructing corresponding 3D trajectories for facial motion classification. In this stereo system, the head rotation can be near $90^{\circ}$. However, a limitation of above studies is inability to separate rigid and non-rigid motion that makes the expression recognition fail to achieve accurate result in real-life image sequences.

The affine model is used in [9] to solve the separation problem, but it can't work for large out-of-plane rotation because of the perspective effects caused by depth variations. The facial expression scheme in [4] applied the 8-parameters perspective projection model and affine model with a curvature function to parameterize large head rotations and non-rigid facial motions, respectively. Facial expression recognition was achieved by assigning different threshold values to the different motion parameters of the models. However, this approach reduces the recognition sensitivity and accuracy of slightly different facial expressions. The work in [5] estimates the camera parameters and reconstructs the corresponding 3D facial geometry of the subject; the 3D pose is recovered by using the Markov Chain Monte Carlo method and each image is warped to frontal-view canonical face image.

Some of above studies [1], [7], [11], [14], [16] recognized the limited six basic expressions, and mouth opening and closing which occurred relatively infrequently in daily life. However, the facial expressions often occurred with changes of more than one feature. Consequently, some other studies [2], [5], [10], [13], [17] used the Facial Action Coding System (FACS) [6] in which the defined action units (AUs) represent the smallest visibly discriminable muscle action and can be combined to create an overall expression. Thus, to separate the rigid and non-rigid motion for facial analysis, we developed a separating system based on the incremental perspective motion model with feature sub-regions. The selected feature sub-regions can take account of recognizing the FACS AUs in the future work.

\section{Incremental Perspective Motion Model}

Computing the perspective transformation between two different view facial images is the main phase of rigid and non-rigid motion separation. Since during the facial expression, large percentage of facial area experienced global motion, the rigid head rotation, while the local facial features, i.e., eyebrows, eyes, nose and mouth, experienced local motion, non-rigid facial expression, obviously. In other words, majority of the face experiencing rigid motion while minority experiencing rigid and non-rigid motions simultaneously. Therefore, the incremental perspective model presented in [12], [8] is used to estimate one global perspective transformation for registering two images under assuming that the interesting region is planar. The used incremental motion model estimates the transformation with considering the major variation caused by the rigid head rotation between images. As for the influence of minor variation that caused by the rigid head rotation and non-rigid facial expression simultaneously, also influenced the transformation estimation but far smaller than the majority.

The contrast between two images may be different because of the change of lightings or camera positions. Since the image registration process need to consider 
the intensity changes of pixels and assume that assuming the pixels of the corresponding positions have the same intensities in different images. Thus, adjusting the contrast of corresponding images beforehand is necessary by using

$$
\begin{aligned}
& I_{1}(x)=\alpha I_{0}(x) \\
& \text { where } \alpha=\left(\frac{\sum I_{1 r}}{\sum I_{0 r}}+\frac{\sum I_{1 g}}{\sum I_{0 g}}+\frac{\sum I_{1 b}}{\sum I_{0 b}}\right) \div 3 \text { in color image } \\
& \text { or } \alpha=\frac{\sum I_{1 g r a y}}{\sum I_{0 \text { gray }}} \text { in gray level image }
\end{aligned}
$$

where $I_{0}$ and $I_{l}$ are two consecutive images, and $\alpha$ is the contrast adjustment between images.

To register two corresponding facial images, $I_{0}(x)$ and $I_{l}\left(x^{\prime}\right)$, with different views, the warping image is computed as: $\tilde{I}_{1}(x)=I_{1}(f(x ; M))$, that is $x^{\prime}$ can be computed by using one parametric motion model $M$ with $x$, i.e. $x^{\prime}=f(x ; M)$. The trick is to find the deformation image, $I_{l}\left(x^{\prime}\right)$, take it into closer registration of $I_{0}(x)$ with bilinear interpolation and then update the parametric motion model $M$. The loop of image warping, registration, and updating parameter can then be iterative computation. To describe the relation between two images by one perspective motion model, these two images are taken as a planar model that the planar perspective transformation warps one image to the other as:

$$
\begin{aligned}
& x^{\prime}=\left[\begin{array}{l}
x^{\prime} \\
y^{\prime} \\
1
\end{array}\right] \cong M x=\left[\begin{array}{lll}
m_{0} & m_{1} & m_{2} \\
m_{3} & m_{4} & m_{5} \\
m_{6} & m_{7} & m_{8}
\end{array}\right]\left[\begin{array}{l}
x \\
y \\
1
\end{array}\right] \\
& \Rightarrow x^{\prime}=\frac{m_{0} x+m_{1} y+m_{2}}{m_{6} x+m_{7} y+m_{8}}, y^{\prime}=\frac{m_{3} x+m_{4} y+m_{5}}{m_{6} x+m_{7} y+m_{8}}
\end{aligned}
$$

To recover the parameters, the transformation matrix $M$ is iteratively updated by using

$$
\begin{aligned}
& M \leftarrow(I+D) M \\
& \text { where } D=\left[\begin{array}{lll}
d_{0} & d_{1} & d_{2} \\
d_{3} & d_{4} & d_{5} \\
d_{6} & d_{7} & d_{8}
\end{array}\right]
\end{aligned}
$$

In equation (3), $D$ represents the deformation (i.e. incremental motion) and is used to update the incremental perspective motion model $M$ (i.e. warping function). That is, resampling (warping) image $I_{l}$ with the transformation $x^{\prime} \cong(I+D) M x$ is equivalent to warping the resampled image $\tilde{I}_{1}$ by $x^{\prime \prime} \cong(I+D) x$, where

$$
x^{\prime \prime}=\frac{\left(1+d_{0}\right) x+d_{1} y+d_{2}}{d_{6} x+d_{7} y+\left(1+d_{8}\right)} \text { and } y^{\prime \prime}=\frac{d_{3} x+\left(1+d_{4}\right) y+d_{5}}{d_{6} x+d_{7} y+\left(1+d_{8}\right)}
$$

To compute the incremental motion parameter vector, $d=\left(d_{0}, \ldots, d_{8}\right)$, the minimizing squared error metric is formulated as: 


$$
\begin{aligned}
E(d) & =\sum_{i}\left[\tilde{I}_{1}\left(x_{i}^{\prime \prime}\right)-I_{0}\left(x_{i}\right)\right]^{2} \\
& \approx \sum_{i}\left[\tilde{I}_{1}\left(x_{i}\right)+\nabla \widetilde{I}_{1}\left(x_{i}\right) \frac{\partial x_{i}^{\prime \prime}}{\partial d} d-I_{0}\left(x_{i}\right)\right]^{2}=\sum_{i}\left[g_{i}^{T} J_{i}^{T} d+e_{i}\right]^{2}
\end{aligned}
$$

where $e_{i}=\tilde{I}_{1}\left(x_{i}\right)-I_{0}\left(x_{i}\right)$ is the intensity (grayvalue) error, $g_{i}^{T}=\nabla \tilde{I}_{1}\left(x_{i}\right)$ is the image gradient of $\tilde{I}_{1}$ at $x_{i}$, and $J_{i}=J_{d}\left(x_{i}\right)$ is the Jacobian of the resampled point coordinate $x_{i}^{\prime \prime}$ with respect to $d$, that is:

$$
J_{d}(x)=\frac{\partial x^{\prime \prime}}{\partial d}=\left[\begin{array}{lllllllll}
x & y & 1 & 0 & 0 & 0 & -x^{2} & -x y & -x \\
0 & 0 & 0 & x & y & 1 & -x y & -y^{2} & -y
\end{array}\right]^{T}
$$

Then the least-squared error problem can be solved through

$$
\begin{aligned}
& \frac{\partial E(d)}{\partial d}=0 \\
& \Rightarrow A d=-b, \text { where } A=\sum_{i} J_{i} g_{i} g_{i}^{T} J_{i}^{T} \text { and } b=\sum_{i} e_{i} J_{i} g_{i}
\end{aligned}
$$

where $A$ is the Hessian matrix and $b$ is the accumulated gradient or residual. Thus, the incremental motion parameter vector, $d$ can be calculated using pseudo inverse as:

$$
d=-\left(A^{T} A\right)^{-1} A^{T} b
$$

To modify the current motion estimate procedure, the computational effort requires to take single gradient descent in parameter space included the three-step computation: (1) the warping of $I_{1}\left(x^{\prime \prime}\right)$ into $\tilde{I}_{1}(x),(2)$ computing the local intensity errors $e_{i}$ and gradients $g_{i}$, and (3) accumulating $e_{i}$ and $g_{i}$ in $A$ and $b$. Because of computing the monomials in $J_{i}$ and the formations in $A$ and $b$, the step (3) is computational expensive. To lower the computational cost, the image is divided into patches $P_{j}$; and make the approximation that $J\left(x_{i}\right)=J_{j}$ is constant within each patch. Thus, equation (7) is rewritten as:

$$
\begin{aligned}
& A \approx \sum_{j} J_{j} A_{j} J_{i}^{T} \text { with } A_{j}=\sum_{i \in P_{j}} g_{i} g_{i}^{T} \\
& \text { and } b \approx \sum_{j} J_{j} b_{j} \text { with } b_{j}=\sum_{i \in P_{j}} e_{i} g_{i}
\end{aligned}
$$

The computation of this patch-based algorithm only needs to evaluate $J_{j}$ and accumulate $A$ and $b$ once per patch. There is the other drawback that gradient descent approach is susceptible to local minimum during the parameter estimation process. To enlarge the convergent region, a milti-resolution approach [3] is used and the estimation result of the incremental motion parameter vector, $d$, at the coarser levels can be used as the initial values at the finer levels. In this work, each image is decomposed into 4 levels from level 0 (the original finest resolution) to level 3 (the coarsest resolution). From level 0 to level 3, the patch-size 8, 4, 2 and 2 are considered at each level, respectively. 


\section{Rigid and Non-rigid Motion Separation System}

In this system, we used the incremental perspective motion model [8] mentioned above to register corresponding sub-regions of two consecutive facial images having different views. Transformation between two images can be taken as a global perspective transformation, i.e. the incremental perspective motion model, $M$, under the assumption that the interesting region is planar. This assumption under facial analysis is that the depth variation of the face is very small compared to the distance between face and camera. That is, the face is almost flat. To make this assumption more general, we take video image sequence as input and consider the transformation between corresponding sub-regions. The positions of the sub-regions were defined in the first frame of sequence by using [15] to automatically locate the facial points.

The parameters of the incremental perspective transformation, $M$, can be used to model the rigid head motion. That is, the parameters of the perspective transformation, $M$, between registered sub-regions can be estimated as the parameters of the warping function. In other words, the rigid and non-rigid motions can be separated by image warping process with warping function $M$. The system flowchart is shown in Fig. 1 and the detailed statement for the separation process is in following contents.

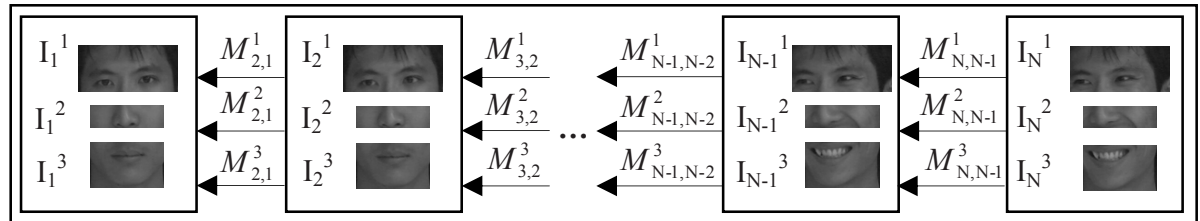

(a). Registering the corresponding sub-regions between $I_{i}$ and $I_{i+1}, i=1 \sim N-1$.

The warping function $M_{i+1, i}^{k}$ : sub-region $k$ warps from $I_{i+1}$ to $I_{i}, k=1 \sim 3$. rigid motion. Each image $I_{i+1}$ is warping frame by frame, and $V_{i+1, j}$ is the warped image of $I_{i+1}$, where $i+1$ is $i+1^{\text {th }}$ image of sequence and $j$ is $j^{\text {th }}$ warped image.
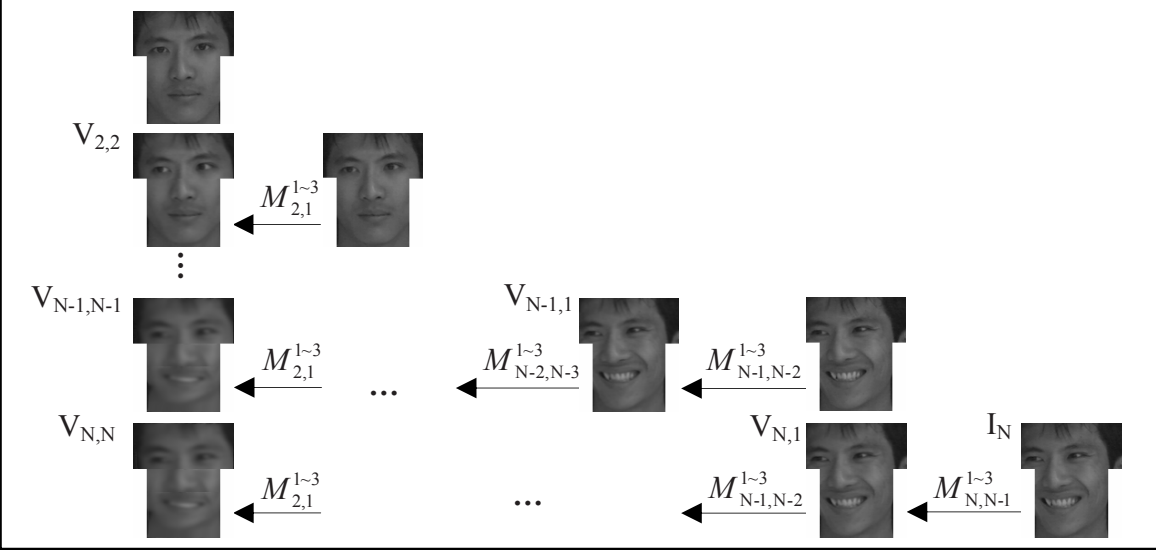

Fig. 1. System flowchart of local incremental perspective motion model 


\subsection{Feature Sub-region Selection}

The transformation estimation takes the major variation between two images as the basis and localizes the minor one. However, to a small extent, the minor variation caused by rigid and non-rigid motions still influences the estimation process. In addition, the depth variation between features, such as eyes and nose, nose and mouth, may violate the assumption that the face is almost flat. Consequently, the sub-regions are used that each contained the specific local feature; in our work three sub-regions, one for the eyebrows and eyes, one for the nose and the other for the mouth, as Fig.1 shown. The area of local facial feature contrasts smaller with area of others of the same sub-region, that is, the majority of the sub-region is not influenced by the facial expression. Each sub-region is independent that the transformation estimations are not influenced by each other. And it is more closed to the assumption that the environment is planar. As Fig. 2 shows that the sub-region selection commences by using the feature point location estimation presented in [15] to automatically locate the feature points in the first frame of sequence. All input image sequences are restricted that subjects commencing with frontal-view and neutral facial expression; that is, the first frame of sequence is supposed to be no rigid and non-rigid motion. The two horizontal lines, $\mathrm{L}_{1} \sim \mathrm{L}_{2}$, and four vertical lines, $\mathrm{L}_{3} \sim \mathrm{L}_{6}$, are decided by points $\mathrm{P}_{1} \sim \mathrm{P}_{6}$; the distances $\mathrm{D}_{1}$ (distance of $\mathrm{P}_{7}$ and $\mathrm{P}_{9}$ ) and $\mathrm{D}_{2}$ (distance of $\mathrm{P}_{8}$ and $\mathrm{P}_{9}$ ) are also be defined. Then, the positions of three sub-regions with respect to the image coordinate can be determined, that the three fixed positions are also used to locate the sub-regions in the remaining frames of sequence.
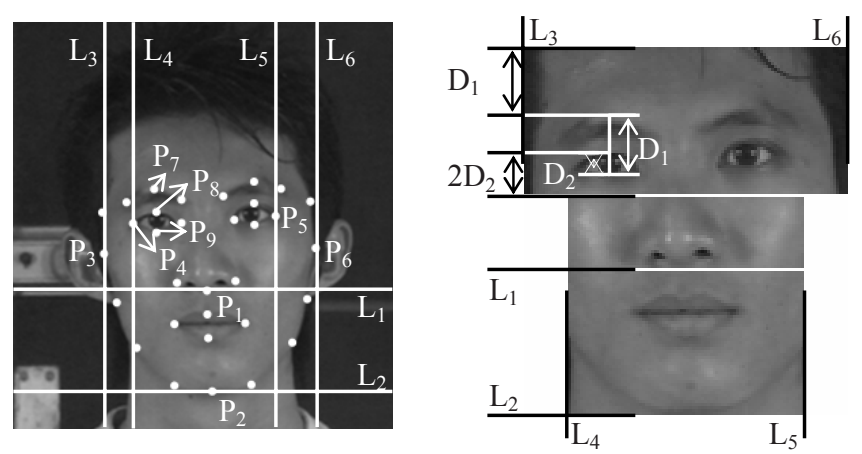

Fig. 2. The automatically located facial feature points and selected sub-regions

\subsection{Incremental Registration and Separation Process for Image Sequence}

For reducing the influence of depth variation between images, the image sequence is taken as the input. After determining the three positions of sub-regions of the input sequence in the first frame, the $N$ images can be divided into $3 N$ sub-regions. Then, the warp functions $M_{i+1, i}^{j}$ of the corresponding sub-regions, there are $3(N-1)$ functions 
in sequence with $N$ images, will be calculated frame by frame using the incremental motion model as shown in Fig.1(a).

Then, Fig.1(b) shows the separation process which also implement frame by frame. Three sub-regions of image $I_{i+1}$ warp to those of image $I_{i}$ by corresponding $M_{i+1, i}^{k}$, $k=1 \sim 3$ as three sub-regions, and its warping image $V_{i+1, l}$, constructed by three subregions, represented image $I_{i+1}$ removing the motion between $I_{i+1}$ and $I_{i}$. If there were outliers occurred, that means the computed corresponding locations don't within the corresponding sub-regions while warping from $I_{i+1}$ to $I_{i}$; we would use the pixellocation of $I_{i+1}$ to take the intensity of corresponding pixel-location of $I_{i}$ for synthesizing $V_{i+1,1}$. Then, the separation process is continued that the warping image $V_{i+1,1}$ is warping to $I_{i-1}$ by $M_{i, i-1}^{k}$ and synthesizing another warping image $V_{i+1,2}$. The process is repeating until the warping image of $I_{i+1}$ warping to $I_{l}$; and the final producing warping image $V_{i+1, i+1}$ shows the result of image $I_{i+1}$ removing the motions between $I_{i+1}$ and $I_{l}$.

\section{Experimental Results}

As shown in Table 1, the testing database contains 50 Asian subjects (35 males and 15 females) that all the subjects did not wear eyeglasses. Each subject performs 3 upper facial expressions, i.e. AU4, AU1+4 and AU1+2, and 6 lower facial expressions, i.e. AU12, AU12+25, AU20+25, AU9+17, AU17+23+24 and AU15+17, with pan rotation. They demonstrated facial expressions without previous training and the sequences were videotaped under constant illumination.

Fig. 3 shows the input image sequence and the separating (warping) results of using the affine model, 8-parameter model, global incremental perspective motion model and our local incremental perspective motion model. For comparing the separating results in the same criterion, each image warped to the template constructed by three sub-regions selected in our approach. These separating results are blurred because of taking bilinear interpolation during the warping process. The subtraction images of input frame 0 represent the differences between the warping result of the current frame and the image at the frame 0 .

Table 1. Testing database. The image size and the average facial size are $320 \times 240$-pixels resolution and $110 \times 125$ pixels, respectively.

\begin{tabular}{|c|c|c|}
\hline Subjects & Upper/ Lower facial expressions & Pan \\
\hline 50 & $3 / 6$ & $0^{\circ} \sim+30^{\circ}$ and $0^{\circ} \sim-30^{\circ}$ \\
\hline
\end{tabular}

Table 2. The average difference rate between the warping image at the current frame and the input frame 0

\begin{tabular}{|c|c|c|c|}
\hline Affine model & 8-parameter model & Global incremental motion model & Our Approach \\
\hline $63.6 \%$ & $73.2 \%$ & $39.4 \%$ & $26.2 \%$ \\
\hline
\end{tabular}




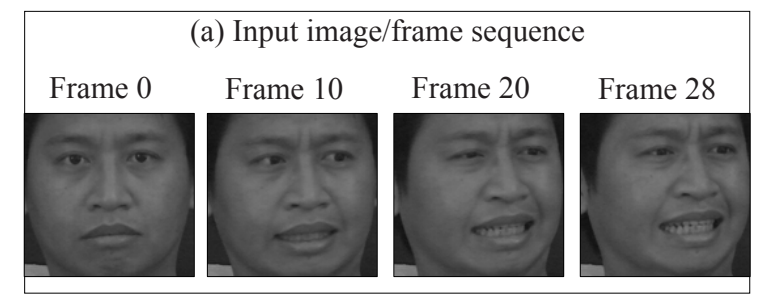

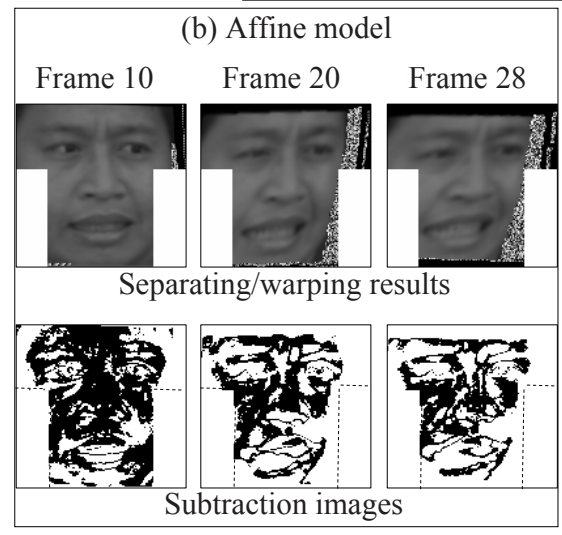

(d) Global incremental motion model

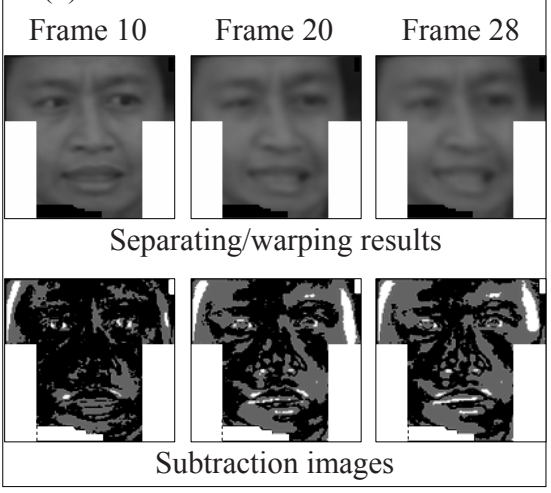

(c) 8-parameter model

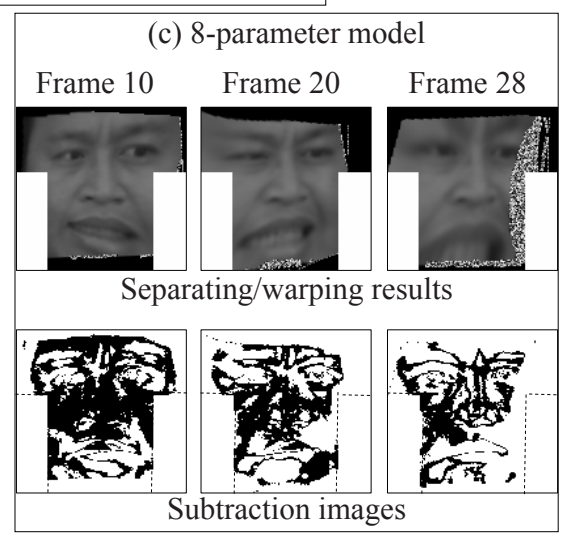

(e) Our approach's results
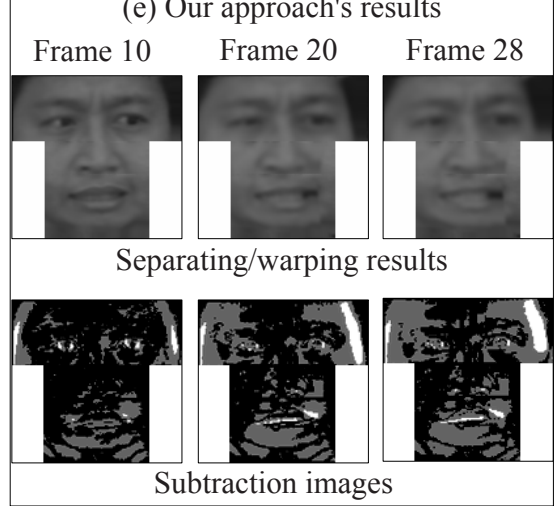

Fig. 3. Separation results of 4 models and subtractions with the first input frame

From Fig. 3, we can find that the separating results of using the affine mode can not take care of the image sequence with out-of-plane motion. It is because the affine model doesn't contain the factors of the depth variations. For the 8-parameter motion model, since parameter estimation process is susceptible to local minimum, so the warping results of the 8-parameter motion model are distorted. In addition, the global incremental perspective motion model is estimated based on the entire facial image that large local motions, i.e. facial expressions, may affect the global transformation estimation as shown in Fig.3.(d). Our results are better than the other three models since the use of sub-regions that local motion within one sub-region wouldn't affect the transformation estimations of the others. As shown in Fig.3.(d) and Fig.3.(e), the 

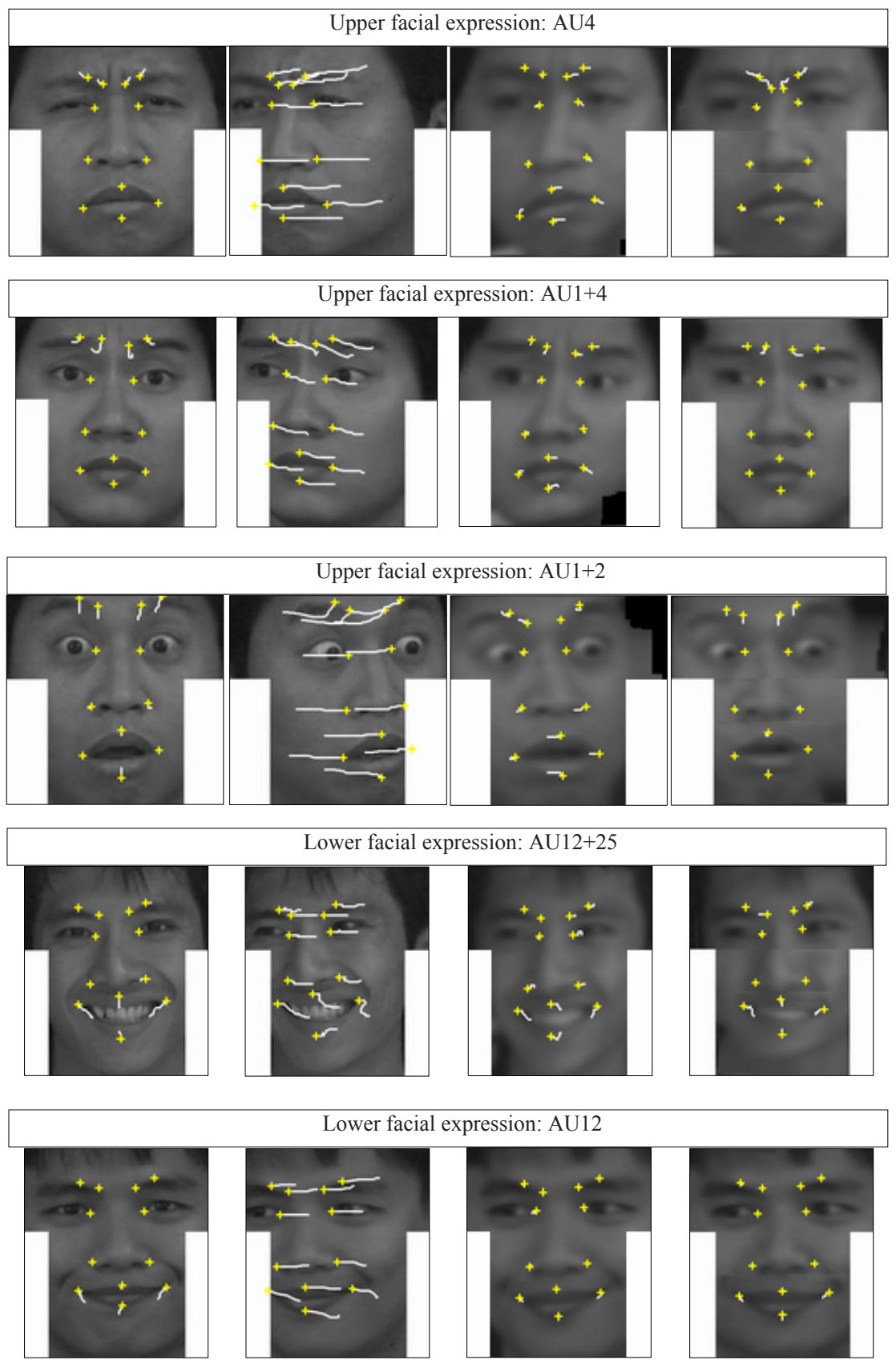

Fig. 4. The lists of nine expressions of the testing database that each expression has four images, frontal-view, side-view, separating result of global incremental motion model and our separating result, from left to right. The "+" represents the position of feature point at last frame. And the trajectory shows the variation of the feature point from the first frame to the last. 

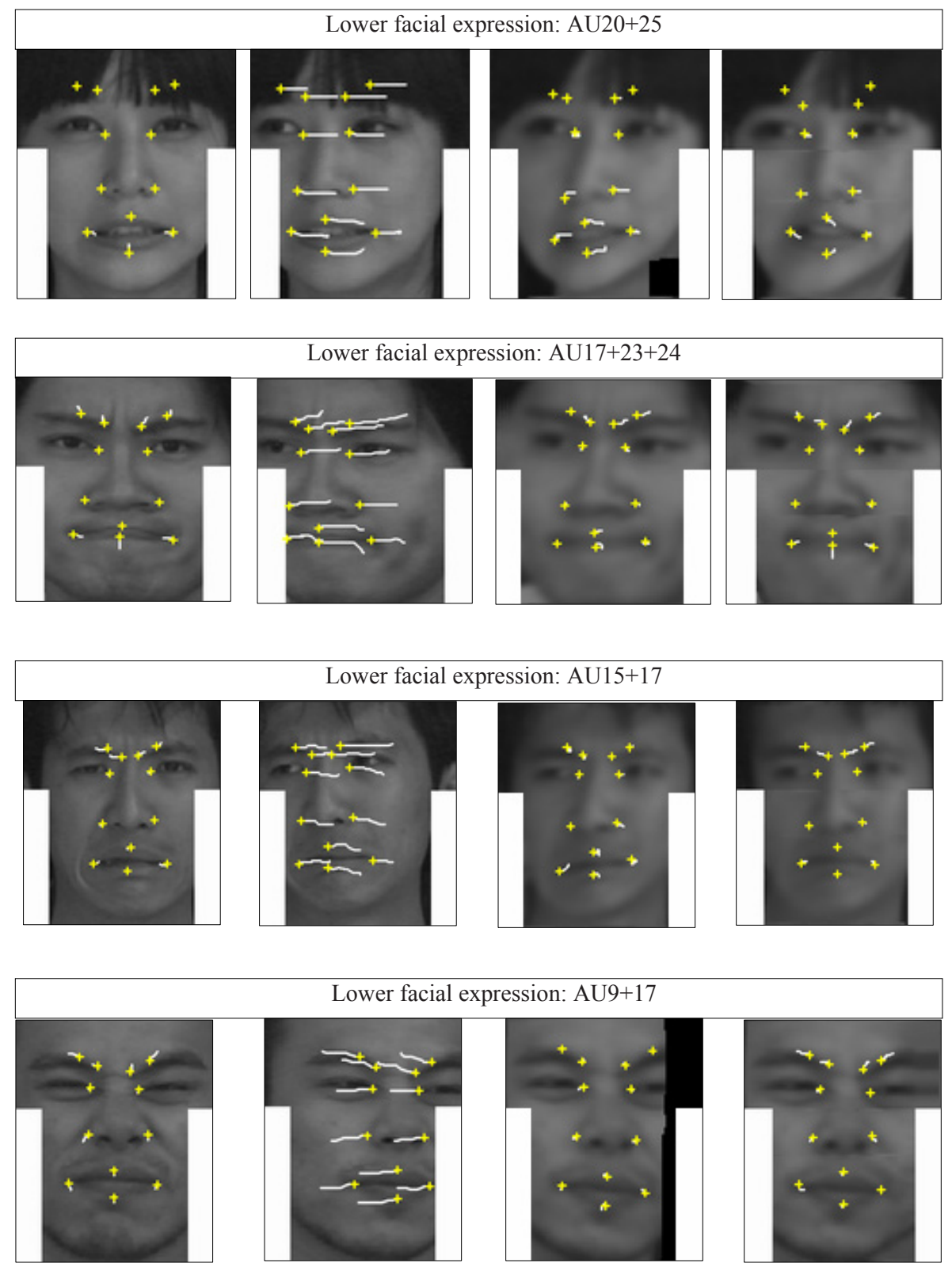

Fig. 4. (continued)

nose region in the global incremental motion model's result is not registered as well as our approach. Table 2 shows the average difference rate of four motion models. The difference rate is the percentage of the ratio between the numbers of the pixels 
whose subtraction grayvalues are above the threshold and the total pixels of warping image. Fig.4 demonstrates the results of our work. The frontal-view image means the last image of the sequence that the subject demonstrated expression without head rotation. The side-view image means the last image of the sequence demonstrated expression with head rotation. The separating result of global incremental motion model and our separating result are the separation of side-view image. For comparison, each image also shows the facial feature points' trajectories that described the variations of the feature points from the first frame to the last. Compared with the trajectories of global incremental motion model's results, our separating results are similar to frontal-view.

\section{Conclusions}

The feature of our approach is estimating the motions between sub-regions with incremental motion model and a multi-resolution approach. The incremental motion model overcomes the limitations of the affine and the 8-parameter perspective projection models in large head rotation angles. By taking account of sub-regions, the influences of depth variation between features are reduced and furthermore the transformation estimations of each sub-region are not affected by other sub-regions. Then, the used multi-resolution approach prevents the parameter estimation get trapped to local minimum. The running time of warping function calculating and image warping are 6.2 seconds and 14.5 seconds, respectively, with 29 frames in the input sequence (Intel Pentium $4 \mathrm{CPU} 3.2 \mathrm{GHz}$ ). The deblurring of warping image and other head rotations as tilt and roll rotations would be considered in future work.

\section{References}

1. Anderson, K., McOwan, P.W.: A Real Time Automated System for the Recognition of Human Facial Expression. IEEE Tran. on SMC 36(1), 96-105 (2006)

2. Bartlett, M.S., Littlewort, G., Frank, M., Lainscsek, C., Fasel, I., Movellan, J.: Fully Automatic Facial Action Recognition in Spontaneous Behavior. In: International Conf. on FG, pp. 223-230 (2006)

3. Bergen, J.R., Anandan, P., Hanna, K.J., Hingorani, R.: Hierarchical model-based motion estimation. In: Proc. of ECCV 1992, pp. 237-252 (May 1992)

4. Black, M., Yacoob, Y.: Recognizing Facial Expressions in Image Sequences Using Local Parameterized Models of Image Motion. IJCV, 23-48 (1997)

5. Braathen, B., Bartlett, M.S., Littlewort, G., Smith, E., Movellan, J.R.: An Approach to Automatic Recognition of Spontaneous Facial Actions. In: International Conf. on FG, pp. 345-350 (2002)

6. Ekman, P., Friesen, W.V.: Facial Action Coding System. Consulting Psychologist Press Inc, San Francisco, CA (1978)

7. Gokturk, S.B., Bouguet, J.Y., Tomasi, C., Girod, B.: Model-Based Face Tracking for View-Independent Facial Expression Recognition. In: International Conf. on FG, pp. 272-278 (2002)

8. Hua, W.: Building Facial Expression Analysis System. CMU Tech. Report (1998) 
9. Lien, J.J., Kanade, T., Cohn, J.F., Li, C.C.: Subtly Different Facial Expression Recognition and Expression Intensity Estimation. In: CVPR, pp. 853-859 (1998)

10. Lucey, S., Matthews, I., Hu, C., Ambadar, Z., de la Torre, F., Cohn, J.: AAM Derived Face Representations for Robust Facial Action Recognition. In: International Conf. on FG, pp. 155-160 (2006)

11. Rosenblum, M., Yacoob, Y., Davis, L.S.: Human Emotion Recognition from Motion Using a Radial Basis Function Network Architecture, Uni. of Maryland, CS-TR-3304 (1994)

12. Szeliski, R., Shum, H.: Creating Full View Panoramic Image Mosaics and Environment Maps. In: Proc. of Siggraph 1997 (August 1997)

13. Tian, Y., Kanade, T., Cohn, J.F.: Evaluation of Gabor-Wavelet-Based Facial Action Unit Recognition in Image Sequences of Increasing Complexity. In: International Conference on FG, pp. 218-223 (2002)

14. De la Torre, F., Yacoob, Y., Davis, L.S.: A Probabilistic Framework for Rigid and Nonrigid Appearance Based Tracking and Recognition. In: International Conf. on FG, pp. 491-498 (2000)

15. Twu, J.T., Lien, J.J.: Estimation of Facial Control-Point Locations. In: IPPR Conf. on Computer Vision, Graphics and Image Processing (2004)

16. Yacoob, Y., Davis, L.S.: Recognizing Human Facial Expressions from Long Image Sequence Using Optical Flow. IEEE Tran. on PAMI 18(6), 636-642 (1996)

17. Zhang, Y., Ji, Q.: Active and Dynamic Information Fusion for Facial Expression Understanding from Image Sequences. IEEE Tran. on PAMI 27, 699-714 (2005) 